\title{
Conserving the lymphatics from the arm using fluorescence imaging in patients with breast cancer at high risk of postoperative lymphedema: a pilot study
}

\author{
Kwang Hyun Yoon ${ }^{1}$, Sung Mook Lim², Bonyong Koo ${ }^{3}$, Jee Ye Kim ${ }^{4}$, Hyung Seok Park ${ }^{4}$, Seho Park ${ }^{4}$, \\ Seung Il Kim ${ }^{4}$, Byeong-Woo Park ${ }^{4}$, Young Up Cho \\ ${ }^{1}$ Department of Surgery, Gangneung Asan Medical Center, University of Ulsan College of Medicine, Gangneung, Republic of Korea; ${ }^{2}$ Department \\ of Surgery, Gangseo MizMedi Hospital, Seoul, Republic of Korea; ${ }^{3}$ Department of Surgery, U\&U Surgery Clinic, Seoul, Republic of Korea; \\ ${ }^{4}$ Division of Breast Surgery, Department of Surgery, Breast Cancer Center, Yonsei Cancer Center, Severance Hospital, Yonsei University College of \\ Medicine, Seoul, Republic of Korea; ${ }^{5}$ Department of Surgery, CHA Ilsan Medical Center, CHA University, Goyang, Republic of Korea \\ Contributions: (I) Conception and design: YU Cho; (II) Administrative support: BW Park; (III) Provision of study materials or patients: KH Yoon, SM \\ Lim; (IV) Collection and assembly of data: B Koo, JY Kim; (V) Data analysis and interpretation: HS Park, S Park, SI Kim; (VI) Manuscript writing: \\ All authors; (VII) Final approval of manuscript: All authors. \\ Correspondence to: Young Up Cho, MD, PhD. Department of Surgery, CHA Ilsan Medical Center, CHA University, Goyang, Republic of Korea. \\ Email: youngup.cho@gmail.com.
}

Backgroundk Postoperative lymphedema in breast cancer survivors is a serious complication that develops from axillary lymph node dissection (ALND), chemotherapy, and radiation therapy. Axillary reverse mapping (ARM) was recently introduced to reduce lymphedema. This pilot study aimed to investigate the feasibility of preserving the ARM node using fluorescence imaging for patients at high risk of lymphedema.

Methods: We prospectively screened patients with breast cancer who had pathologic node-positive disease at diagnosis and were scheduled for neoadjuvant chemotherapy (NCT). The sentinel lymph node (SLN) was identified using blue dye and radioisotope, while the ARM node was traced using indocyanine green (ICG). In cases in which SLN was negative on the intraoperative frozen section examination, the ARM node and lymphatics were preserved.

Results: Of the 20 screened patients, six whose metastatic axillary lymph node (ALN) was converted to clinically node-negative disease after NCT were enrolled. No patients experienced recurrence at 24 months postoperative. Four patients who had a preserved ARM node did not develop lymphedema. One patient whose ARM node was not preserved due to SLN identification failure did not develop postoperative lymphedema. One patient who underwent ALND without ARM node conservation because of metastatic SLN on frozen section examination developed postoperative lymphedema.

Conclusions: ARM is oncologically safe, decreases the incidence of postoperative lymphedema, and allows for the early detection of postoperative lymphedema in patients who underwent ALND. Ultimately, ARM may help improve the quality of life of patients with pathologic node-positive breast cancer.

Koywords: Axillary reverse mapping (ARM); breast neoplasm; fluorescence imaging; indocyanine green (ICG); lymphedema

Submitted Aug 21, 2019. Accepted for publication Feb 28, 2020.

doi: 10.21037 /gs.2020.03.29

View this article at: http://dx.doi.org/10.21037/gs.2020.03.29 


\section{Introduction}

Axillary lymph node (ALN) status is the most important prognostic factor for patients with breast cancer (1). Sentinel lymph node biopsy (SLNB) was developed to evaluate ALN metastasis and prevent complications following axillary lymph node dissection (ALND) (2). SLNB is the standard modality used to assess the ALN metastatic burden of patients with clinical node-negative breast cancer. SLNB is possible in patients with clinically node-negative disease at the time of surgery after neoadjuvant chemotherapy (NCT) and even in patients with cytologically proven node-positive disease before treatment (3-5).

The improved survival rate of patients with breast cancer has led to an increased quality of life. Lymphedema diminishes the quality of life of breast cancer survivors. Postoperative lymphedema develops from various factors and treatment modalities including ALND, radiation therapy, chemotherapy, postoperative infection, and higher body mass index (BMI) (6). Several methods to reduce lymphedema have been developed and evaluated. Among such procedures, axillary reverse mapping (ARM) has been evaluated in various studies (7-11). One study reported that postoperative ipsilateral lymphedema occurs due to lymph stasis resulting from impaired lymphatic drainage from the arm during axillary surgery (11). The ARM node is detected via mapping of the lymphatic drainage from the ipsilateral arm at the axillary area. The ARM technique is indicated to prevent or detect postoperative lymphedema at the early stage through preservation of the ARM node.

Several mapping techniques are available to identify the lymph node including blue dye and radioisotope. Several recent studies reported using the mapping method with indocyanine green (ICG) (12-14). ICG injected into the interstitial tissue is taken up by the lymphatics (15), and fluorescence imaging allows for visualization of the subcutaneous lymphatic pathway. ICG mapping is reportedly safe and useful and an alternative modality to blue dye or radioisotope mapping (16).

The risk of lymphedema markedly increases in patients who undergo ALND, radiation therapy, and chemotherapy. Thus, the clinical challenge is reducing the incidence of lymphedema or detecting it at an early stage, before the refractory phase, in patients at high risk of this complication. Therefore, we hypothesized that if histologically proven node-positive disease is converted to clinically node-negative disease at the time of surgery and the SLN is negative on the frozen section examination, complete ALND can be omitted. Moreover, preserving the ARM node reduces postoperative lymphedema, even in those who underwent ALND. This pilot study aimed to investigate the feasibility of preserving the ARM node using fluorescence imaging for patients at high risk of lymphedema.

\section{Methods}

\section{Patient selection}

We prospectively screened patients with clinical $\mathrm{N} 1(\mathrm{f})$ breast cancer at diagnosis who underwent NCT between March and July 2017 at Severance Hospital (Seoul, South Korea). The clinical category of the primary tumor and regional lymph node was assessed according to the American Joint Committee on Cancer Staging Manual eighth edition (17). The exclusion criteria were stage IV or clinical T4 or clinical N2 or higher breast cancer at diagnosis. NCT regimens were chosen by the physician according to clinical stage, hormonal receptor status, and human epidermal growth factor receptor 2 (HER2) according to the American Society of Clinical Oncologists/College of American Pathologist guidelines (18). The molecular subtypes included luminal A-like, luminal B-like, HER2-positive, and triple-negative according to the European Society for Medical Oncology Clinical Practice guideline (19). The primary tumor response to NCT was evaluated according to the response criteria of the Japanese Breast Cancer Society (20). The ALN response to NCT was defined as disappearance of the metastatic lymph node on ultrasonography and magnetic resonance imaging after preoperative image studies. The breast cancer response to NCT was sequentially assessed using physical examination, mammography, ultrasonography, and magnetic resonance imaging. Finally, the patients with suspicious ALN findings, a palpable enlarged lymph node, loss of the fatty hilum, cortical thickening $>3 \mathrm{~mm}$, or markedly hypoechoic cortex after NCT were excluded.

\section{Ethical statement}

This study was approved by the Institutional Review Board of Severance Hospital, Yonsei University Health System (2018-2590-001). Written informed consent was obtained from all patients prior to study participation.

\section{Mapping technique}

The SLN was traced using radioisotopes and blue dye 
A

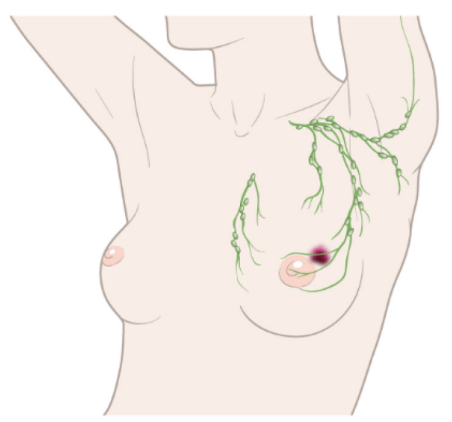

C

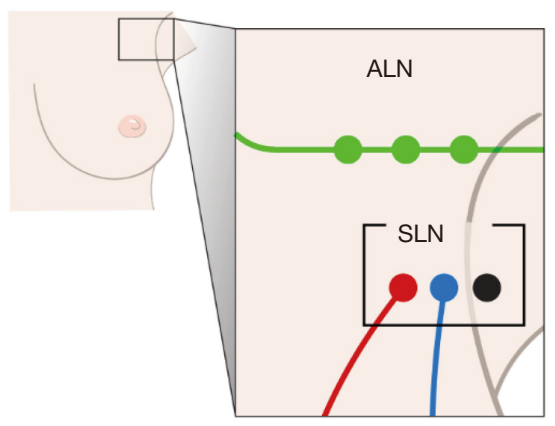

B

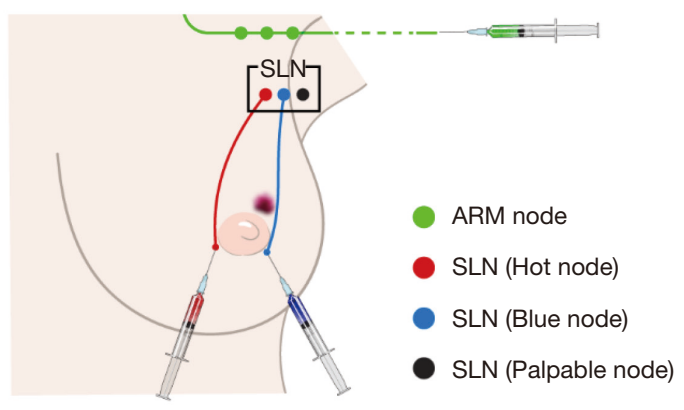

E
D

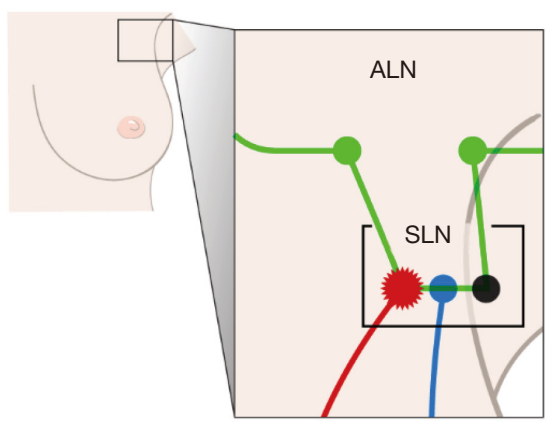

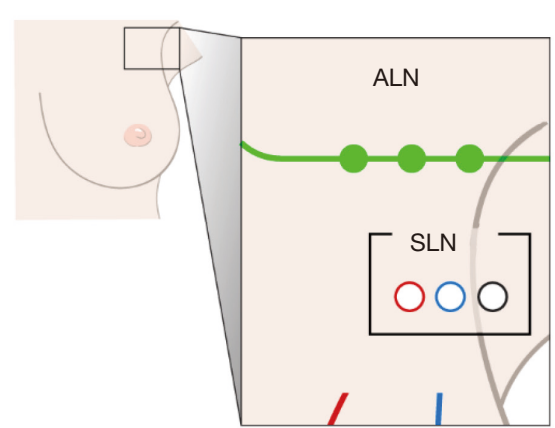

Figure 1 Mapping technique of SLN and ARM node. Manual for ALN surgery in select cases. (A) Anatomy of breast and ALN; (B) mapping technique of SLN and ARM node; (C) SLN-ARM node non-crossover and node-negative disease confirmed via frozen section examination; (D) SLN-ARM node crossover or node-positive disease confirmed via frozen-section examination; (E) SLN identification failure. SLN, sentinel lymph node; ARM, axillary reverse mapping; ALN, axillary lymph node.

(Figure 1). The ARM node was traced using ICG. We diluted $0.5 \mathrm{mCi} 99 \mathrm{mTc}$-Phytate (Korea Atomic Energy Research Institute, Daejeon, Korea) in $0.5 \mathrm{~mL}$ of saline and injected it into the areolar subcutaneous layer in the direction of the primary tumor at the Department of Nuclear Medicine immediately prior to the surgery. After the induction of general anesthesia, $0.8 \%$ indigo carmine dye (Carmine, Korea United Pharm Inc., Seoul, Korea) was injected at four points around the areola, and $0.5 \mathrm{mg} / \mathrm{mL}$ ICG (Allencare, Seoul, Korea) was diluted in $1 \mathrm{~mL}$ saline and injected into the ipsilateral medial aspect of the wrist and the second webspace of the hand. The SLN was defined as a hot, blue, and palpable node. Nodes $>10 \%$ of the most radioactive node on a handheld gamma probe (Neoprobe Gamma Detection System, Neoprobe Corp., Dublin, OH, USA) were identified as hot nodes. Blue nodes and any lymph nodes at the end of a blue lymphatic channel were considered to be SLNs. Clinically suspicious palpable nodes in the surgical field were also resected as SLNs. ARM nodes were visualized under near-infrared fluorescence imaging with SPY Elite ${ }^{\circledR}$ (Novadaq Technologies, Inc., Bonita Springs, FL, USA).

\section{Axillary surgery}

All axillary surgeries were performed through separate axillary incisions. After dividing the clavipectoral fascia, we traced the ARM node and lymphatics from the ipsilateral arm using a fluorescence camera. These procedures were repeated during axillary surgery. First, each SLN was excised in the axillary cavity based on the hot, blue, palpable node. Each SLN was then sent for frozen-section analysis. If an SLN was negative, we performed ALND with preservation of the ARM node and lymphatics. Meanwhile, if an SLN was positive or not traced, we performed ALND without preserving the ARM node and lymphatics. ALND was performed according to standard criteria. Postoperative clinical follow-up was scheduled at regular 6-month intervals or performed whenever the patients had subjective abnormal symptoms including arm swelling, heaviness, firmness, tightness, and limited arm movement.

\section{Lymphedema}

The arm circumference was measured during the 
Table 1 Clinicopathologic characteristics of the patients

\begin{tabular}{|c|c|c|c|c|c|c|c|c|c|}
\hline Patient no. & Age & $\mathrm{BMI}$ & NCT & Breast surgery & ALN RTx. & Pathologic TNM stage & ER & PR & HER2 \\
\hline 2 & 57 & 19.31 & $\mathrm{AC} \rightarrow \mathrm{wP}+\mathrm{H}$ & BCS & $(+)$ & ypTONO & $(-)$ & $(-)$ & 3 \\
\hline 3 & 35 & 21.03 & TCHP & BCS & $(+)$ & ypT1bN1 & $(+)$ & $(+)$ & 3 \\
\hline 4 & 59 & 29.64 & $\mathrm{AC} \rightarrow \mathrm{wP}$ & BCS & $(+)$ & ypTONO & $(-)$ & $(-)$ & 0 \\
\hline 6 & 52 & 21.09 & $\mathrm{AC} \rightarrow \mathrm{wP}$ & TM & $(+)$ & ypT1aNo & $(-)$ & $(-)$ & 0 \\
\hline
\end{tabular}

Hormone receptor status and HER2 gene expression were evaluated via core needle biopsy prior to the NCT. BMI, body mass index; NCT, neoadjuvant chemotherapy; ALN, axillary lymph node; RTx., radiation therapy; TNM, tumor, node, metastasis; ER, estrogen receptor; PR, progesterone receptor; HER2, human epidermal growth factor receptor 2; AC, anthracycline followed by cyclophosphamide; wP, weekly paclitaxel; H, trastuzumab; BCS, breast-conserving surgery; TM, total mastectomy; CHP, docetaxel/ carboplatin/trastuzumab/pertuzumab.

preoperative period and every 6 months. Patients who had subjective abnormal symptoms in the ipsilateral arm or abnormal findings on a self-check were instructed to visit the hospital for an arm circumference assessment. At the Department of Rehabilitation Medicine, a clinical lymphologist evaluated the arm status via physical examination and limb circumference measurement using a flexible non-stretch ruler. Portions of the hand (thumb, thena, hypothena, and superior metacarpal), wrist, and 5 -cm increments starting at the folding line at elbow flexion from the wrist to the axilla were measured. The criterion for diagnosis of lymphedema was a circumference $\geq 2 \mathrm{~cm}$ compared with that of the contralateral limb based on the International Society of Lymphology guideline (21). Patients with a circumferential difference $<2 \mathrm{~cm}$ of the bilateral limbs or no abnormal finding on expert physical examination underwent sequential circumferential arm measurements and lymphoscintigraphy. Abnormal findings on lymphoscintigraphy included dermal backflow, collateral lymphatic vessels, and decreased lymphatic uptake.

\section{Results}

\section{Patient characteristics}

Of the 20 patients who were screened, six were included in our pilot study. The detailed patient characteristics are shown in Table 1. The median age was 57 (range, 52-67) years at the time of treatment, and the mean follow-up duration was 24 months. All patients had invasive ductal carcinoma. The median breast tumor size was 2.1 (range, 1.4-3.2) $\mathrm{cm}$ on ultrasonography at the time of diagnosis. Three patients had HER2-positive disease, while the other three had triple-negative breast cancer. All patients who underwent total mastectomy also underwent radiation therapy at the chest wall, level III lymph node, internal mammary lymph node, and supraclavicular lymph. Meanwhile, those who underwent breast-conserving mastectomy underwent radiation therapy of the entire breast, tumor bed, level III lymph node, internal mammary lymph node, and supraclavicular lymph node. Three patients with both a primary tumor and node positivity achieved a pathologic complete response (pCR) to NCT.

\section{$A L N$}

An SLN (hot and/or blue) was detected in five patients. The identification rate of SLN using the dual method was $83.3 \%$ (5/6). The median number of retrieved SLN and total ALN $(\mathrm{SLN}+$ non-SLN + ARM node; only cases in which the ARM node was not preserved) were 1.5 (range, $0-2$ ) and 6.5 (range, 4-11), respectively (Table 2). Only one patient was confirmed to have a metastasis in the ALN including the SLN and non-sentinel ALN. The metastatic foci of ALN was measured to be 3 and $4 \mathrm{~mm}$ on permanent section by a specialist in breast pathology. Furthermore, this metastatic SLN connected with the ARM node in the axillary cavity (Figure 2). The nodal pCR rate to NCT was $83.3 \%$. In cases of SLN mapping failure, the SLN could not be traced using the dual method; however, the lymphatic pathway from the ipsilateral arm was visualized on fluorescence imaging. This patient underwent ALND without preservation of the ARM node. Two patients did not achieve ARM node preservation because the SLN was not identified and because of 
Table 2 Removed axillary lymph node status and result of surgery

\begin{tabular}{|c|c|c|c|c|c|c|c|c|}
\hline $\begin{array}{l}\text { Patient } \\
\text { no. }\end{array}$ & $\begin{array}{l}\text { ALN count } \\
\text { (mets/total) }\end{array}$ & $\begin{array}{l}\text { SLN (hot/blue/ } \\
\text { mets/count) }\end{array}$ & $\begin{array}{c}\text { Non-sentinel ALN } \\
\text { (mets/total) }\end{array}$ & $\begin{array}{l}\text { ARM node (flu/ } \\
\text { mets/count) }\end{array}$ & $\begin{array}{l}\text { SLN-ARM node } \\
\text { (crossover) }\end{array}$ & $\begin{array}{l}\text { ARM node } \\
\text { (preserving) }\end{array}$ & Lymphoscintigraphy & Lymphedema \\
\hline 1 & $0 / 7$ & (Hot/blue/0/2) & $0 / 5$ & (Flu/?/?) & $(-)$ & $(+)$ & No abnormal findings & $(-)$ \\
\hline 3 & $2 / 10$ & (-/blue/1/2) & $1 / 8$ & (Flu/1/1) & $(+)$ & $(-)$ & Not done & $(+)$ \\
\hline 4 & $0 / 4$ & (Hot/blue/0/2) & $0 / 2$ & (Flu/?/?) & $(-)$ & $(+)$ & No abnormal findings & $(-)$ \\
\hline 6 & $0 / 11$ & (Hot/blue/0/1) & $0 / 10$ & (Flu/?/?) & $(-)$ & $(+)$ & Not done & $(-)$ \\
\hline
\end{tabular}

ALN, axillary lymph node; ARM, axillary reverse mapping; Flu, fluorescence lymph node; Mets, metastatic lymph node; SLN, sentinel lymph node.
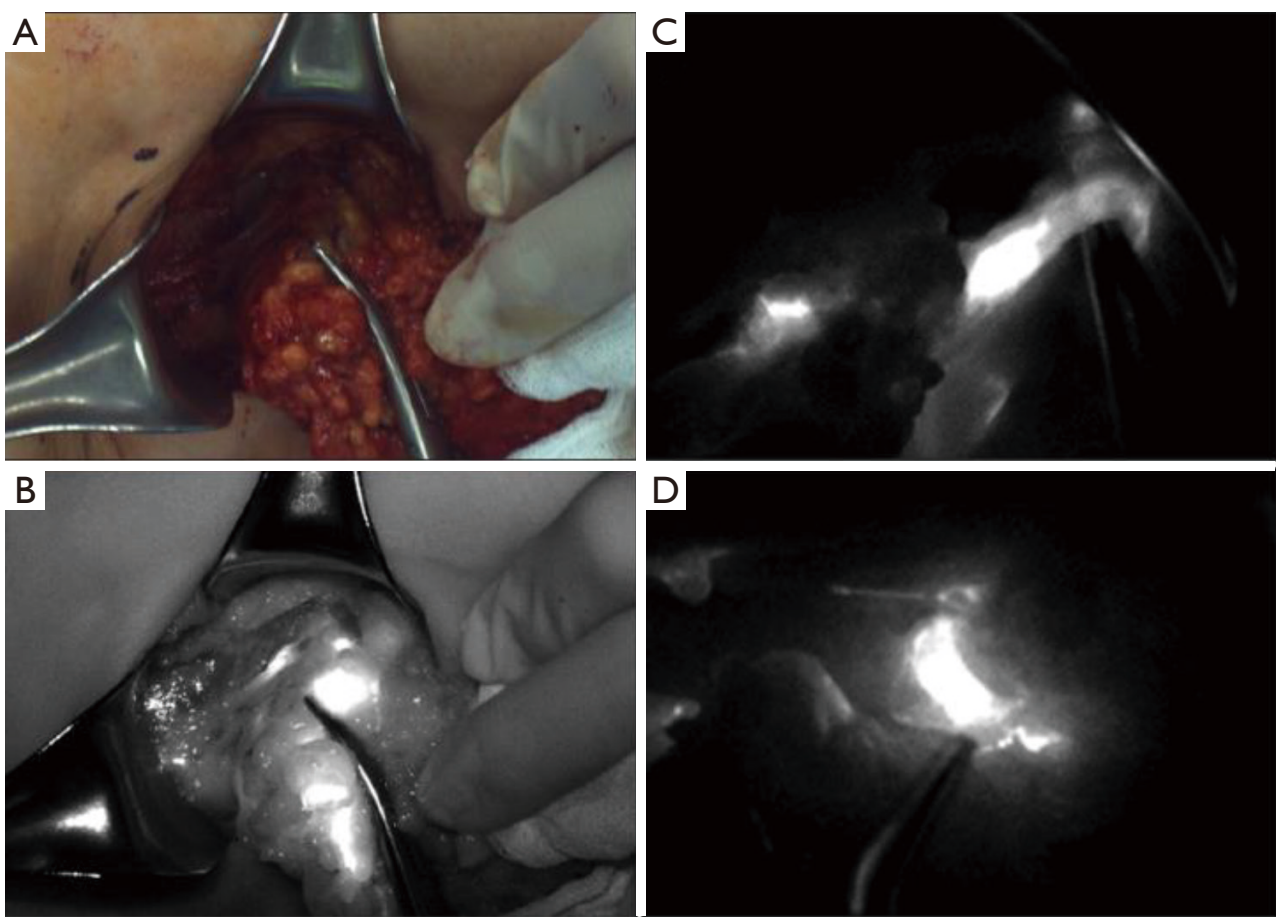

Figure 2 Identification of ARM node and lymphatics from the ipsilateral arm using fluorescence imaging. (A) Intraoperative photo of SLNARM node crossover; (B) fluorescence image of SLN-ARM node crossover; (C) identification of the ARM node and pathway during SLN resection; (D) preserving the ARM node and lymphatics during ALND. ARM, axillary reverse mapping; SLN, sentinel lymph node; ALND, axillary lymph node dissection.

SLN-ARM node crossover. No patient developed regional recurrence during the 24-month follow-up.

\section{Lymphedema}

Three patients had subjective symptoms in the ipsilateral arm. Of them, two underwent evaluations of the lymphatic pathway of the ipsilateral arm via lymphoscintigraphy because the arm circumference did not fulfill the lymphedema criteria. No abnormal findings were noted on lymphoscintigraphy. One other patient was diagnosed with grade I lymphedema. She underwent ALND without preservation of the ARM node owing to SLN metastasis and SLN-ARM node crossover. Only one of the two patients 
who underwent ALND without ARM node preservation developed lymphedema.

\section{Discussion}

In our pilot study, we evaluated the mapping technique for identifying the lymphatic channel from the ipsilateral arm via ICG injection and verified the technical feasibility of preserving the ARM node and lymphatics through near-infrared fluorescence imaging for patients who are candidates for ALND.

Patients with node-positive breast cancer at diagnosis receive more aggressive treatment than those with nodenegative disease. These treatments, including ALND, radiation therapy, and systemic chemotherapy, are associated with postoperative ipsilateral lymphedema. For oncologic safety, ALND is pursued in some patients who undergo immediate surgery after the pathologic confirmation of ALN metastasis. However, complete ALND can be skipped in some patients with clinical node-negative disease before surgery who underwent NCT following the diagnosis of node-positive disease during the pretreatment period (22).

A retrospective multicenter analysis compared the disease-free and overall survival between patients who underwent ALND based on the SLNB result and those who underwent complete ALND without SLNB after NCT (23). The SLNB-guided decision group had higher rates of axillary recurrence than the complete ALND group with marginal significance (hazard ratio, 2.33; $\mathrm{P}=0.061$ ). The higher regional recurrence rate in the SLNBguided decision group might have been induced by the lower diagnostic performance of SLNB in previous NCT patients. Furthermore, several clinical trials reported the performance of SLNB for patients with confirmed nodepositive disease at diagnosis who underwent NCT (3-5). The overall false-negative rate of SLNB was $14.2 \%$ in the SENTinel NeoAdjuvant study, $12.6 \%$ in the American College of Surgeons Oncology Group Z1071 (Alliance) trial, and $13.3 \%$ in the Sentinel Node Biopsy Following Neoadjuvant Chemotherapy trial. The diagnostic performance of SLNB was improved via tattooing or clipping before NCT in patients with confirmed positive ALN (24). If tattooing or clipping was not performed for positive ALN before NCT, the false-negative rate of SLNB exceeded $10 \%$. Furthermore, long-term followup results are needed to clarify the diagnostic performance and oncological safety of SLNB in these cases. To ensure superior diagnostic accuracy and oncologic safety, aggressive axillary surgery should be considered until the benefits of SLNB for these patients is clarified.

Several studies reported that the ARM technique has some limitations $(12,25,26)$ including the possibility of a decreased detection rate of SLN or ARM node if only one mapping material is used. Furthermore, the rate of ARM node identification differs between SLNB and ALND, which indicates that the ARM pathway is located on a deeper layer than the SLNB tract or that the transit times of the blue dye through the ARM lymphatics is not suitable for identifying ipsilateral arm lymphatics. Furthermore, in a previous NCT response study, the SLNB accuracy and pCR to NCT for cytologically proven node-positive disease with triplenegative breast cancer were $97.1 \%$ and $58.8 \%$, respectively, using only the radioisotope mapping method (27). If the diagnostic performance is enhanced via the dual mapping method including radioisotope and blue dye, ALND with ARM node preservation might be acceptable diagnostic modalities. However, it is possible that ARM node metastasis, ARM node crossover with SLN, and false negative result of SLNB frozen section examination occurred $(8,10)$. Therefore, for oncologic safety, extended axillary surgery with ARM node preservation should be performed in selective patients with advanced nodal status at diagnosis. The eligibility criteria for this surgery are clinical node-negative disease after NCT, node-negative disease confirmed via intraoperative frozen section examination, and no connection between the SLN pathway and ARM pathway in the axillary cavity. Complete ALND or axillary radiotherapy should be considered for some patients with a false-negative SLNB result on a frozen section examination.

In conclusion, triple mapping techniques can help identify the SLN and ARM nodes and assess their correlation and metastatic burden. The ARM node-preserving technique might decrease the rate of postoperative lymphedema in patients at high risk of this complication. Appropriate prevention techniques, early diagnosis, and treatment are crucial to improve the quality of life of patients who develop post-treatment complications. Clinical trials designed to identify treatment modalities yielding lower complications while providing optimal effect are needed. Ultimately, the ARM technique may help improve the quality of life of patients with pathologic node-positive breast cancer.

\section{Acknowledgments}

The authors thank Medical Illustration \& Design, a part of the Medical Research Support Services of Yonsei University 
College of Medicine, for artistic support related to this work.

Funding: None.

\section{Footnote}

Conflicts of Interest: All authors have completed the ICMJE uniform disclosure form (available at http://dx.doi. org/10.21037/gs.2020.03.29). The authors have no conflicts of interest to declare.

Ethical Statement: The authors are accountable for all aspects of the work in ensuring that questions related to the accuracy or integrity of any part of the work are appropriately investigated and resolved. This study was approved by the Institutional Review Board of Severance Hospital, Yonsei University Health System (2018-2590-001). Written informed consent was obtained from all patients prior to study participation.

Open Access Statement: This is an Open Access article distributed in accordance with the Creative Commons Attribution-NonCommercial-NoDerivs 4.0 International License (CC BY-NC-ND 4.0), which permits the noncommercial replication and distribution of the article with the strict proviso that no changes or edits are made and the original work is properly cited (including links to both the formal publication through the relevant DOI and the license). See: https://creativecommons.org/licenses/by-nc-nd/4.0/.

\section{References}

1. Yoshihara E, Smeets A, Laenen A, et al. Predictors of axillary lymph node metastases in early breast cancer and their applicability in clinical practice. Breast 2013;22:357-61.

2. Veronesi U, Paganelli G, Galimberti V, et al. Sentinel-node biopsy to avoid axillary dissection in breast cancer with clinically negative lymph-nodes. Lancet 1997;349:1864-7.

3. Kuehn T, Bauerfeind I, Fehm T, et al. Sentinel-lymphnode biopsy in patients with breast cancer before and after neoadjuvant chemotherapy (SENTINA): a prospective, multicentre cohort study. Lancet Oncol 2013;14:609-18.

4. Boughey JC, Suman VJ, Mittendorf EA, et al. Sentinel lymph node surgery after neoadjuvant chemotherapy in patients with node-positive breast cancer: the ACOSOG Z1071 (Alliance) clinical trial. JAMA 2013;310:1455-61.

5. Boileau JF, Poirier B, Basik M, et al. Sentinel node biopsy after neoadjuvant chemotherapy in biopsy-proven node- positive breast cancer: the SN FNAC study. J Clin Oncol 2015;33:258-64.

6. DiSipio T, Rye S, Newman B, et al. Incidence of unilateral arm lymphoedema after breast cancer: a systematic review and meta-analysis. Lancet Oncol 2013;14:500-15.

7. Thompson M, Korourian S, Henry-Tillman R, et al. Axillary reverse mapping (ARM): a new concept to identify and enhance lymphatic preservation. Ann Surg Oncol 2007;14:1890-5.

8. Tummel E, Ochoa D, Korourian S, et al. Does axillary reverse mapping prevent lymphedema after lymphadenectomy? Ann Surg 2017;265:987-92.

9. Sakurai T, Endo M, Shimizu K, et al. Axillary reverse mapping using fluorescence imaging is useful for identifying the risk group of postoperative lymphedema in breast cancer patients undergoing sentinel node biopsies. J Surg Oncol 2014;109:612-5.

10. Foster D, Choy N, Porter C, et al. Axillary reverse mapping with indocyanine green or isosulfan blue demonstrate similar crossover rates to radiotracer identified sentinel nodes. J Surg Oncol 2018;117:336-40.

11. Nos C, Lesieur B, Clough KB, et al. Blue dye injection in the arm in order to conserve the lymphatic drainage of the arm in breast cancer patients requiring an axillary dissection. Ann Surg Oncol 2007;14:2490-6.

12. Noguchi $M$, Miura $S$, Morioka $E$, et al. Is axillary reverse mapping feasible in breast cancer patients? Eur J Surg Oncol 2015;41:442-9.

13. Hirche C, Murawa D, Mohr Z, et al. ICG fluorescenceguided sentinel node biopsy for axillary nodal staging in breast cancer. Breast Cancer Res Treat 2010;121:373-8.

14. Sugie T, Kassim KA, Takeuchi M, et al. A novel method for sentinel lymph node biopsy by indocyanine green fluorescence technique in breast cancer. Cancers (Basel) 2010;2:713-20.

15. Benya R, Quintana J, Brundage B. Adverse reactions to indocyanine green: a case report and a review of the literature. Cathet Cardiovasc Diagn 1989;17:231-3.

16. Jung SY, Kim SK, Kim SW, et al. Comparison of sentinel lymph node biopsy guided by the multimodal method of indocyanine green fluorescence, radioisotope, and blue dye versus the radioisotope method in breast cancer: a randomized controlled trial. Ann Surg Oncol 2014;21:1254-9.

17. Amin MB, Edge S, Greene F, et al. AJCC Cancer Staging Manual. 8th ed. New York: Springer, 2017.

18. Wolff AC, Hammond ME, Hicks DG, et al. Recommendations for human epidermal growth factor 
receptor 2 testing in breast cancer: American Society of Clinical Oncology/College of American Pathologists clinical practice guideline update. J Clin Oncol 2013;31:3997-4013.

19. Senkus E, Kyriakides S, Ohno S, et al. Primary breast cancer: ESMO Clinical Practice Guidelines for diagnosis, treatment and follow-up. Ann Oncol 2015;26 Suppl 5:v8-30.

20. Kurosumi M, Akashi-Tanaka S, Akiyama F, et al. Histopathological criteria for assessment of therapeutic response in breast cancer (2007 version). Breast Cancer 2008; 15:5-7.

21. Executive Committee. The Diagnosis and Treatment of Peripheral Lymphedema: 2016 Consensus Document of the International Society of Lymphology. Lymphology 2016;49:170-84.

22. Gradishar WJ, Anderson BO, Balassanian R, et al. Invasive Breast Cancer Version 1.2016, NCCN Clinical Practice Guidelines in Oncology. J Natl Compr Canc Netw 2016;14:324-54.

23. Kang YJ, Han W, Park S, et al. Outcome following

Cite this article as: Yoon KH, Lim SM, Koo B, Kim JY, Park HS, Park S, Kim SI, Park BW, Cho YU. Conserving the lymphatics from the arm using fluorescence imaging in patients with breast cancer at high risk of postoperative lymphedema: a pilot study. Gland Surg 2020;9(3):629-636. doi: 10.21037/ gs.2020.03.29 sentinel lymph node biopsy-guided decisions in breast cancer patients with conversion from positive to negative axillary lymph nodes after neoadjuvant chemotherapy. Breast Cancer Res Treat 2017;166:473-80.

24. Park S, Koo JS, Kim GM, et al. Feasibility of charcoal tattooing of cytology-proven metastatic axillary lymph node at diagnosis and sentinel lymph node biopsy after neoadjuvant chemotherapy in breast cancer patients. Cancer Res Treat 2018;50:801-12.

25. Noguchi M. Axillary reverse mapping for breast cancer. Breast Cancer Res Treat 2010;119:529-35.

26. Han C, Yang B, Zuo WS, et al. The feasibility and oncological safety of axillary reverse mapping in patients with breast cancer: a systematic review and meta-analysis of prospective studies. PLoS One 2016;11:e0150285.

27. Park S, Park JM, Cho JH, et al. Sentinel lymph node biopsy after neoadjuvant chemotherapy in patients with cytologically proven node-positive breast cancer at diagnosis. Ann Surg Oncol 2013;20:2858-65. 